\title{
An Estimation of the Burden of Sports Injuries among African Adolescents
}

\author{
Drake G. LeBrun ${ }^{1,2, *}$, Julius del Rosario ${ }^{3}$, John D. Kelly ${ }^{4}$, Sherry M. Wren 5 , David A. Spiegel ${ }^{6}$, \\ Nyengo Mkandawire, ${ }^{7,8}$ Richard A. Gosselin ${ }^{9}$, Adam L. Kushner ${ }^{10,11,12}$ \\ ${ }^{1}$ Department of Epidemiology, Harvard T.H. Chan School of Public Health, 677 Huntington Avenue, Boston, MA 02115, USA \\ ${ }^{2}$ Perelman School of Medicine, University of Pennsylvania, 3400 Civic Center Blvd., Jordan Medical Education Center, Philadelphia, PA 19104, USA \\ ${ }^{3}$ School of Engineering and Applied Science, University of Pennsylvania, 220 South 33rd Street, 107 Towne Building, Philadelphia, PA 19104, USA \\ ${ }^{4}$ Division of Sports Medicine, Department of Orthopaedic Surgery, Perelman School of Medicine, University of Pennsylvania, 235 S 33rd St, Philadelphia, PA 19104, USA \\ ${ }^{5}$ Department of Surgery, Stanford University, School of Medicine, 300 Pasteur Dr, Stanford, CA 94305, USA \\ ${ }^{6}$ Department of Orthopaedic Surgery, Children's Hospital of Philadelphia, 3401 Civic Center Blvd., Philadelphia, PA 19104, USA \\ ${ }^{7}$ Department of Orthopaedic Surgery, University of Malawi, College of Medicine, P/B 360, Chichiri, Blantyre 3, Malawi \\ ${ }^{8}$ Flinders University School of Medicine, Sturt Rd, Bedford Park, South Australia 5042, Australia \\ ${ }^{9}$ Institute of Global Orthopaedics and Traumatology, Department of Orthopaedic Surgery, University of California San Francisco, $255023 r d$ Street, \\ Building 9, 2nd Floor, San Francisco, CA 94110, USA \\ ${ }^{10}$ Department of Surgery, Columbia University College of Physicians and Surgeons, 177 Fort Washington Ave., New York, NY 10032, USA \\ ${ }^{11}$ Department of International Health, Johns Hopkins Bloomberg School of Public Health, 615 N. Wolfe Street, Baltimore, MD 21205, USA \\ ${ }^{12}$ Surgeons OverSeas, 99 Avenue B, Suite 5E, New York, NY 10009, USA
}

\section{ARTICLE INFO}

Article History

Received 16 March 2017

Accepted 25 October 2017

Keywords

Sports

injury

adolescent

Africa

\begin{abstract}
The extent to which sports injuries contribute to the burden of injury among adolescents in low- and middle-income countries (LMICs) is unknown. The goal of this study was to estimate the incidence of sports injuries among adolescents in Africa. Data from the World Health Organization Global School-Based Student Health surveys were used to estimate the annual number of African adolescents sustaining sports injuries. Gender-stratified injury rates were calculated and applied to every African country's adolescent population to estimate country-specific and continent-wide injury totals. A total of 21,858 males and 24,691 females from 14 countries were included in the analysis. Country-specific annual sports injury rates ranged from $13.5 \%$ to $38.1 \%$ in males and $5.2 \%$ to $20.2 \%$ in females. Weighted average sports injury rates for males and females were $23.7 \%$ (95\% CI $23.1 \%-24.2 \%$ ) and $12.5 \%$ ( $95 \%$ CI 12.1\%-12.9\%), respectively. When these rates were extrapolated to the adolescent populations of the African continent, an estimated 15,477,798 (95\% CI 15,085,955-15,804,333) males and 7,943,625 (95\% CI 7,689,429-8,197,821) females sustained sports injuries. Our findings suggest that over 23 million African adolescents sustained sports injuries annually. Further work will help to more precisely define the burden of sports injuries in LMICs and the role that surgery can play in mitigating this burden.
\end{abstract}

(C) 2018 Atlantis Press International B.V. This is an open access article under the CC BY-NC license (http://creativecommons.org/licences/by-nc/4.0/).

\section{INTRODUCTION}

Adolescent health is a pressing yet neglected issue in global health policy [1,2]. Investing in adolescent health can have longterm impacts on adult health, quality of life, and economic productivity $[3,4]$. Injuries comprise a major portion of the global burden of disease among adolescents $[1,2,5]$. Injuries sustained while playing sports - whether organized or non-organized recreational activities - are extremely common in this population [6]. However, the extent to which sports injuries contribute to the global burden of injury is poorly documented. Large schoolbased cohort studies, injury databases, and surveillance systems have provided some information on the incidence of sports injuries in high-income countries (HICs) [7-12], but comparable

\footnotetext{
* Corresponding author.Email:Drake.Lebrun@uphs.upenn.edu
}

studies from low- and middle-income countries (LMICs) are lacking $[6,13]$.

Given the paucity of information on sports injuries in LMICs, especially those affecting adolescents, we sought to estimate the burden of sports injuries among adolescents in Africa using existing survey data from schoolchildren in several African nations. Estimating the burden of sports injuries across countries and regions is uniquely challenging given the wide country-to-country variability in types of sports played, the existence of organized versus non-organized sports, and different rates of sports participation across gender, age, and socioeconomic strata. Furthermore, it is important to note that there are virtually no high-quality data on sports injuries in LMICs; therefore, despite significant methodological limitations, the work presented here may serve to guide further studies to better describe and quantify the true burden of sports injuries in Africa and other LMICs. 


\section{MATERIALS AND METHODS}

\subsection{Description of Survey and Study Population}

This study relied on publicly available data from the World Health Organization (WHO) Global School-Based Health Survey (GSHS) (accessible at http://www.who.int/chp/gshs/en/). The GSHS is a school-based surveillance project whose aim is to assess behavioral risk factors and protective factors among young people aged 13-17 years old. The survey methodology has been extensively described elsewhere [14]. To summarize, survey data were collected via a twostage cluster sample design. Schools within each surveyed country were initially selected with a probability proportional to their reported enrollment size. Classes were then randomly selected and all students in selected classes were eligible to participate. Selfadministered questionnaires were completed during one classroom period under the supervision of trained survey administrators.

\subsection{Data Collection}

For the purposes of this study, only GSHS data on unintentional injuries and violence were considered. Specifically, data on two questions were abstracted. For the first question, study participants were asked, "During the past 12 months, how many times were you seriously injured?" A "serious injury" was defined as an injury that caused the child to miss at least one full day of usual activities (such as school, sports, or a job) or required treatment by a doctor or nurse. Participants were able to indicate either "no times," "one time," "two times," continuing up to "twelve or more times." For the second question, study participants were asked, "During the past 12 months, what were you doing when the most serious injury happened?" Eight options were provided, including: "playing or training for a sport" and "walking or running, but not as part of playing or training for a sport." Of note, this question only referred to the "most serious" injury among all the possible serious injuries sustained by each child. Thus, it is possible that a child's sports injury would be unrepresented by this question if it were not the most severe injury sustained over a one year period. Although these questions were the same across surveys whenever they were posed, some country surveys did not ask questions related to injury.

Survey codebooks from all available African nations were reviewed to identify whether surveys collected data on injury etiology. We included all surveys that collected data on serious injuries and their etiologies and excluded those that did not. In cases of multiple surveys conducted over different years, the most recent survey was used.

We obtained raw data files from every included African country. Data on the numbers of males and females sustaining at least one serious injury, and the subset of those that were attributable to sports, were abstracted. Individuals who did not report gender were excluded from analysis. Country-specific sports injury rates - the proportion of "most serious" injuries attributable to sports - were calculated and stratified by gender. These rates were then weighted by the sample size of each survey to obtain a sample-weighted continent-wide estimate for both male and female adolescents. These estimates were then extrapolated to the male and female populations aged 13-17 for each country in
Africa. Population data were obtained from the United Nations Population Division 2015 estimates.

\subsection{Statistical Analysis}

Descriptive statistics were used to present sample characteristics. A Pearson's chi-square test was used to determine associations between gender and injury rates among each sample. All tests were two-sided tests with a level of significance of $\alpha \leq 0.05$. Data analyses were performed using STATA 14.1 (StataCorp, College Station, TX).

\section{RESULTS}

\subsection{Overview of Surveys}

GSHS data were available from 22 African countries (Algeria, Benin, Botswana, Djibouti, Egypt, Ghana, Kenya, Libya, Malawi, Mauritania, Mauritius, Morocco, Namibia, Senegal, Seychelles, Sudan, Swaziland, Tanzania, Tunisia, Uganda, Zambia, and Zimbabwe). Of these, 14 included data on serious injuries attributable to sports (Botswana, Djibouti Egypt, Ghana, Kenya, Libya, Mauritius, Morocco, Swaziland, Tanzania, Tunisia, Uganda, Zambia, and Zimbabwe). The surveys in Africa were conducted over the years 2003-2014. Survey data from these countries represented a total of 21,858 males and 24,691 females, with an additional 888 individuals excluded for not reporting gender (Fig. 1).

\subsection{Sports Injury Rates}

Gender-stratified sports injury rates for the 14 included countries are summarized in Table 1. The percentage of individuals sustaining at least one serious sports injury ranged from $13.5 \%$ to $38.1 \%$ for males and $5.2 \%$ to $20.2 \%$ for females. Males were more likely than females to sustain at least one serious sports-related injury in 13 of the 14 sampled countries $(\mathrm{P}<.01$ for all but one country). Weighted average rates of sustaining at least one serious sports injury for males and females were $23.7 \%$ (95\% CI $23.1 \%-24.2 \%$ ) and $12.5 \%$ (95\% CI $12.1 \%-12.9 \%)$, respectively. When these rates were applied to the adolescent populations of every African country, an estimated $15,477,798(95 \%$ CI 15,085,955-15,804,333) males and 7,943,625 (95\% CI 7,689,429-8,197,821) females in Africa sustained at least one serious sports injury per year. In total, an estimated 23,423,181 (95\% CI 22,546,829-24,353,815) adolescents in Africa sustained at least one sports injuries over a one-year period. A sensitivity analysis was also performed by excluding countries with surveys prior to 2007 and found no significant differences in the injury estimates.

\section{DISCUSSION}

In this modeling study based on WHO GSHS survey data, we estimated that over 23 million African adolescents between the ages of 13 and 17 years old sustained at least one serious sports injury over a one-year period. Although this study is purely descriptive, it quantifies a significant burden of disease due to sports injury and 


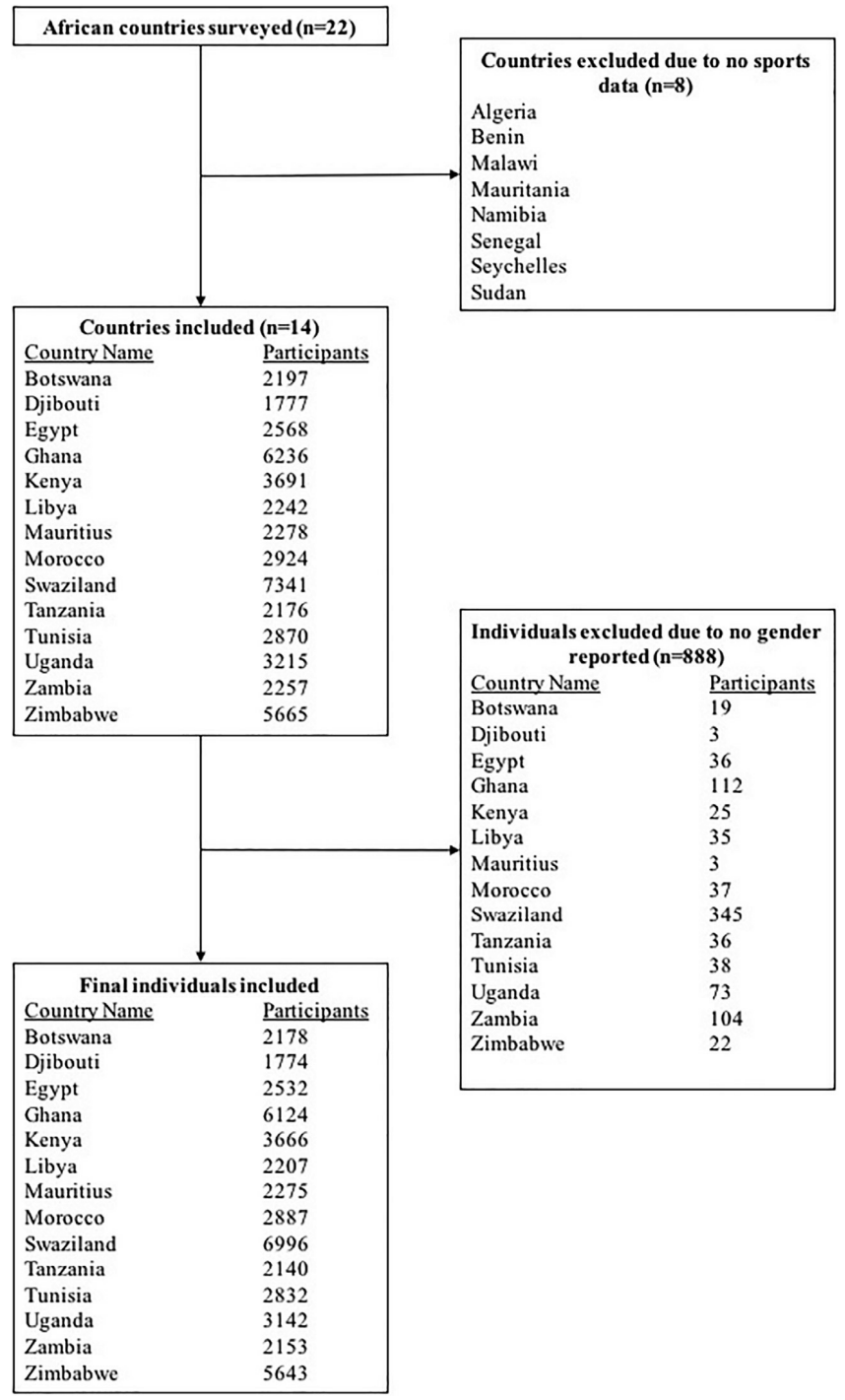

Figure 1 Study flowchart demonstrating numbers of countries and participants included highlights an area where additional research is needed to more precisely define this burden.

Our findings highlight three key points. First, the injury incidence due to sports among African adolescents is high, with over 23 million adolescents sustaining at least one serious sports injury over a one-year period. This is consistent with evidence from past studies demonstrating that sports are the most common cause of injury in adolescents in LMICs and HICs [14,15]. Second, injury frequency varies significantly between males and females. In fact, the weighted average sports rates of at least one serious sports injury among males were nearly twice the rates among females $(23.8 \%$ versus $12.4 \%$ ). Moreover, males were significantly more likely than females to sustain at least one sports injury in all but one of the surveyed countries. This is consistent with another study that found that boys had a higher all-cause injury rate in 40 of 47 LMICs [6]. Third, injury rates vary widely between countries. For example, in Tanzania, the rates for males and females were $13.5 \%$ and $9.8 \%$ while comparable rates in Uganda were $30.4 \%$ and 18.0 , respectively.

The nature of sports injuries in LMICs is likely different from that in HICs. One study of adolescent injury in four LMICs across three continents suggested that sports-related injuries in LMICs are unique from sports injuries in HICs because they are influenced by poor infrastructure (e.g., children playing sports on uneven terrain may get injured more easily) and compounded risks (e.g., roads are common places for children to play non-organized sports in LMICs, thereby increasing the risk of motor vehicle accidents) [16]. Additionally, due to the limited availability of appropriate and timely medical care, the impact of a given sports injury in a lowincome country may be more severe than that in a high-income country. For example, a tear of the anterior cruciate ligament (ACL) a common injury among adolescents in competitive sports often requires surgical repair and extended knee immobilization, followed by physical therapy [10]. Without appropriate surgery and follow-up, the injured adolescent may become permanently disabled, thus less able to attend school and/or provide needed labor to support a family [17].

Table 1 Overview of included surveys and gender-stratified numbers of adolescents sustaining at least one sports injury over a one year period

\begin{tabular}{|c|c|c|c|c|c|c|}
\hline Country & $\begin{array}{l}\text { Survey } \\
\text { year }\end{array}$ & $\begin{array}{l}\text { Total number of } \\
\text { surveyed males }\end{array}$ & $\begin{array}{l}\text { Total number of } \\
\text { surveyed females }\end{array}$ & $\begin{array}{l}\text { Percentage of males sustaining } \\
\text { at least one sports injury }\end{array}$ & $\begin{array}{c}\text { Percentage of females sustaining } \\
\text { at least one sports injury }\end{array}$ & $P$ value \\
\hline Botswana & 2005 & 979 & 1199 & $25.5(22.8-28.4)$ & $17.6(15.5-19.9)$ & $<.0001$ \\
\hline Djibouti & 2007 & 1013 & 761 & $20.6(18.2-23.3)$ & $14.7(12.3-17.4)$ & .001 \\
\hline Egypt & 2011 & 1182 & 1350 & $38.1(35.3-40.9)$ & $15.2(13.3-17.2)$ & $<.0001$ \\
\hline Ghana & 2007 & 3207 & 2917 & $28.8(27.2-30.4)$ & $20.2(18.7-21.7)$ & $<.0001$ \\
\hline Kenya & 2003 & 1784 & 1882 & $22.6(20.7-24.6)$ & $16.2(14.6-17.9)$ & $<.0001$ \\
\hline Libya & 2007 & 948 & 1259 & $19.5(17.0-22.2)$ & $7.8(6.4-9.4)$ & $<.0001$ \\
\hline Mauritius & 2007 & 1118 & 1157 & $22.9(20.5-25.5)$ & $7.6(6.1-9.3)$ & $<.0001$ \\
\hline Morocco & 2010 & 1514 & 1373 & $20.3(18.3-22.5)$ & $5.2(4.1-6.5)$ & $<.0001$ \\
\hline Swaziland & 2003 & 2526 & 4470 & $19.7(18.2-21.3)$ & $6.9(6.2-7.7)$ & $<.0001$ \\
\hline Tanzania & 2014 & 1026 & 1114 & $13.5(11.5-15.8)$ & $9.8(8.1-11.7)$ & .007 \\
\hline Tunisia & 2008 & 1379 & 1453 & $18.5(16.5-20.6)$ & $6.1(4.9-7.5)$ & $<.0001$ \\
\hline Uganda & 2003 & 1615 & 1527 & $30.4(28.2-32.7)$ & $18.0(16.1-20.0)$ & $<.0001$ \\
\hline Zambia & 2014 & 1052 & 1101 & $22.0(19.5-24.6)$ & $19.4(17.1-21.9)$ & .149 \\
\hline Zimbabwe & 2014 & 2515 & 3128 & $22.7(21.1-24.4)$ & $13.0(11.9-14.2)$ & $<.0001$ \\
\hline Total & & 21858 & 24691 & $23.7(23.1-24.2)^{\star}$ & $12.5(12.1-12.9)^{\star}$ & \\
\hline
\end{tabular}

${ }^{*}$ Calculated as weighted averages based on relative sample sizes of country surveys. 
Our study has multiple limitations. The GSHS surveys do not capture type of sport played, type of injury, how the injury was managed, or type of school (e.g., public, private, urban, rural, etc.). They also represent a limited sample: of over 50 diverse African countries with different socioeconomic backgrounds, schooling patterns, and sports practices, GSHS surveys were performed in 22 countries and only 14 of those reported data on injuries attributable to sports. The remaining 8 surveyed countries did not collect any information on sports-attributable injuries. Eastern Africa and Northern Africa are relatively well represented compared to West Africa and Central Africa. Additionally, the numbers of students surveyed in each country are not proportional to the adolescent populations of the countries or their respective rates of school attendance, so these surveys may not be representative of the entire countries. The survey period is also wide from 2003 to 2014 - so injury results from different time periods may not be directly compatible due to geopolitical and socioeconomic changes over time. In summary, consolidating these data into an aggregate pool to create sample-weighted sports injury rates carries significant risk of oversimplification of a complex estimation; however, the limited available data precluded the use of a more rigorous method of modeling incidence rates such as multivariable Poisson regression based on multiple relevant covariates. Therefore, the results in this study serve as a first step in better understanding the burden of sports injuries among adolescents in Africa.

Our study may also be subject to information bias. In the WHO GSHS survey, the question assessing whether an injury was due to sports was: "What were you doing when you were injured?" It is also important to note that the survey relied on a student's recall over one year, even though longer recall periods are associated with a higher rate of underestimation and misreporting of serious injuries [18]. Additionally, some students may overreport or underreport injuries due to a variety of social factors.

Due to the nature of the WHO GSHS surveys being conducted among schoolchildren aged 13-17, our study may be subject to selection bias. Specifically, if school attendance is associated with a different risk of injury compared to not attending school, then selection bias may affect the validity and generalizability of our findings. Moreover, school attendance may also be associated with a different risk of playing sports which may further contribute to selection bias.

Lastly, our study should not be interpreted as an estimate of cumulative incidence or incidence rate. These datasets report whether a given child experienced at least one serious injury, with the most serious being attributable to sports. They do not report the number of injuries sustained within a given year and do not account for the possibility of multiple injuries. Moreover, they do not report the cause of every serious injury; rather, they only report the cause of the most serious injury.

Although this study is subject to substantial methodological limitations, it is important to note that there are virtually no high-quality data that describes the extent of sports injuries in African countries or other low-income regions. This study serves as an important initial step that can guide additional studies assessing the burden of sports injuries in LMICs. The objective of this study is not to guide resource allocation or policy recommendations; rather, it is to highlight the lack of useful data on sports injuries and the need for additional studies with more rigorous data.

\section{CONCLUSIONS}

Our study sought to estimate the number of adolescents in Africa sustaining at least one serious sports-related injury over a one year period. Despite methodological limitations, our study suggests that over 23 million African adolescents experience a sport-related injury on a yearly basis. These findings highlight the absence of useful data on sports injury epidemiology in Africa and provide a foundation upon which additional research can be performed.

\section{ACKNOWLEDGEMENT}

None.

\section{REFERENCES}

[1] Mokdad AH, Forouzanfar MH, Daoud F, Mokdad AA, El Bcheraoui C, Moradi-Lakeh M, et al. Global burden of diseases, injuries, and risk factors for young people's health during 19902013: a systematic analysis for the Global Burden of Disease Study 2013. Lancet 2016;6736;1-19.

[2] Burden Global of Disease Pediatrics Collaboration, Kyu HH, Pinho C, Wagner JA, Brown JC, Bertozzi-Villa A, et al. Global and national burden of diseases and injuries among children and adolescents between 1990 and 2013: Findings from the global burden of disease 2013 study. JAMA Pediatr 2016;98121;1-21.

[3] Lassi Z, Salam R, Das J, Wazny K, Bhuta Z. An unfinished agenda on adolescent health: opportunities for interventions. Semin Perinatol 2015;39;353-60.

[4] Resnick M, Catalano R, Sawyer S, Viner R, Patton G. Seizing the opportunities of adolescent health. Lancet 2012;379;1564-7.

[5] Chandran A, Hyder AA, Peek-Asa C. The global burden of unintentional injuries and an agenda for progress. Epidemiol Rev $2010 ; 32 ; 110-20$

[6] Street EJ, Jacobsen KH. Injury incidence among middle school students aged 13-15 years in 47 low-income and middle-income countries. Inj Prev 2015.

[7] Rechel JA, Collins CL, Comstock RD. Epidemiology of injuries requiring surgery among high school athletes in the United States, 2005 to 2010. J Trauma 2011;71;982-9.

[8] Schneider S, Seither B, Tönges S, Schmitt H. Sports injuries: population based representative data on incidence, diagnosis, sequelae, and high risk groups. Br J Sports Med 2006;40;334-9; discussion 339.

[9] Ingram JG, Fields SK, Yard EE, Comstock RD. Epidemiology of knee injuries among boys and girls in US high school athletics. Am J Sports Med 2008;36;1116-22.

[10] Parkkari J, Pasanen K, Mattila VM, Kannus P, Rimpelä A. The risk for a cruciate ligament injury of the knee in adolescents and young adults: a population-based cohort study of 46500 people with a 9 year follow-up. Br J Sports Med 2008;42;422-6. 
[11] Caine D, Maffulli N, Caine C. Epidemiology of injury in child and adolescent sports: injury rates, risk factors, and prevention. Clin Sports Med 2008;27;19-50.

[12] Darrow CJ, Collins CL, Yard EE, Comstock RD. Epidemiology of severe injuries among United States high school athletes: 20052007. Am J Sport Med 2009;37;1798-805.

[13] Khan KM, Thompson AM, Blair SN, Sallis JF, Powell KE, Bull FC, et al. Sport and exercise as contributors to the health of nations. Lancet 2012;380;59-64.

[14] Peltzer K. Injury and social determinants among in-school adolescents in six African countries. Inj Prev 2008;14;381-8.

[15] Pickett W, Dostaler S, Craig W, Janssen I, Simpson K, Shelley $\mathrm{SD}$, et al. Associations between risk behavior and injury and the protective roles of social environments: an analysis of 7235 Canadian school children. Inj Prev 2006;12;87-92.

[16] Morrow V, Barnett I, Vujcich D. Understanding the causes and consequences of injuries to adolescents growing up in poverty in Ethiopia, Andhra Pradesh (India), Vietnam and Peru: a mixed method study. Health Policy Plan 2014;29;67-75.

[17] Farshad M, Gerber C, Meyer DC, Schwab A, Blank PR, Szucs T. Reconstruction versus conservative treatment after rupture of the anterior cruciate ligament: cost effectiveness analysis. BMC Health Serv Res 2011;11;317.

[18] Mock C, Acheampong F, Adjei S, Koepsell T. The effect of recall on estimation of incidence rates for injury in Ghana. Int J Epidemiol 1999;28;750-5. 